\title{
Clinical Problems in Community Mental Health Care for Patients with Severe Borderline Personality Disorder
}

\author{
B. Koekkoek • B. van Meijel • A. Schene • \\ G. Hutschemaekers
}

Received: 16 June 2008/Accepted: 6 October 2009/Published online: 22 October 2009

(c) The Author(s) 2009. This article is published with open access at Springerlink.com

\begin{abstract}
The objective of this research was to assess the problems that professionals perceive in the community mental health care for patients with severe borderline personality disorder that do not fit into specialized therapy. A group of national experts $(n=8)$ participated in a fourphase Delphi-procedure to identify and prioritize the problems. A total of 36 problems reflecting five categories was found: patient-related, professional-related, interaction-related, social system-related, and mental health carerelated. Problems with attachment and dependency and social issues were important patient problems while a lack of skills was an important professional problem. Support from the patient's social system and the mental health system were identified as limited, which resulted in both the patient and the professional feeling isolated. Patient, professional, and organisational characteristics of community
\end{abstract}

B. Koekkoek $(\bowtie)$

Department of Outpatient Community Care, Altrecht Mental

Health Care, Oude Arnhemseweg 260, 3705 BK Zeist,

The Netherlands

e-mail: b.koekkoek@altrecht.nl

B. Koekkoek - G. Hutschemaekers

Institute for Professionalization, Gelderse Roos Mental Health Care, Wolfheze, The Netherlands

B. van Meijel

Research Group Mental Health Nursing, InHolland University of Applied Sciences, Amsterdam, The Netherlands

A. Schene

Department of Psychiatry, Academic Medical Center

of the University of Amsterdam, Amsterdam, The Netherlands

G. Hutschemaekers

Academic Centre of Social Sciences, Radboud University,

Nijmegen, The Netherlands care differ substantially from those of specialized care. The field is thus in need of a more tailored approach that takes these differences into account.

Keywords Community mental health services . Long-term care - Borderline personality disorder . Delphi technique $\cdot$ Focus group

\section{Introduction}

Progress in the psychosocial treatment of borderline personality disorder (BPD) has been made over the last decades. Evidence from randomized clinical trials now provides support for dialectical behavior therapy (Linehan et al. 1991, 2006), mentalization-based therapy (Bateman and Fonagy 1999, 2001, 2008), schema-focused therapy (Giesen-Bloo et al. 2006), transference-focused psychotherapy (GiesenBloo et al. 2006), and supportive therapy (Clarkin et al. 2007). Despite the demonstrated effectiveness of so many therapies for patients with a BPD, not all patients are in a position to reap its benefits. The reasons for this may be the severity of the disorder, defined as: the presence of overly disruptive behavior, uncontrollable anger, aggression, complicating co-morbidity, a lack of emotional stability to attend sessions regularly, social problems that interfere with proper treatment, a lack of motivation, and/or limited intelligence. Our own experience with referral to and working with specialized treatment programs is that many patients with a BPD are simply not indicated for specialized treatment, do not enter, drop out, or fail to improve. In all of the aforementioned treatments, professionals typically try to address the described obstructions in one way or another yet still not all patients fit into these therapies. These treatments also tend to be short as opposed to long term, which means 
that patients must return to regular psychiatric care when still in need of care (e.g., Bateman and Fonagy 2008).

As difficult as it is to assess severity in BPD patients (Tyrer 2005), for most of the patients described above community mental health care provides a safety net. However, the focus of community mental health care is mostly on patients with schizophrenia and other psychotic disorders, which means that it does not really meet "the needs of individuals with BPD, which could account for poor treatment compliance and subsequent hospitalization" (Lieb et al. 2004, p. 455). The professionals in community mental health care are mostly nurses, social workers, occupational therapists, and other non-psychotherapeutically trained professionals (Keown et al. 2002; Hunter et al. 2002; Greenwood et al. 2000; Pomeroy and Ricketts 1985; Lesage and Cope 1988) who often do not know how to provide proper care for patients with severe BPD.

The observed lack of knowledge regarding the care for patients with a severe BPD is not surprising as, despite the fact that many professionals are familiar with them, they only rarely appear in the research literature (e.g., Nehls and Diamond 1993, in this journal), in community care training courses, or in discussions during scientific conferences (Koekkoek et al. 2010). The high rate of attempted suicide (Paris 2002) and the intensive use of psychiatric services (Comtois et al. 2003; Bender et al. 2001) by such patients is noted, but proper care and treatment strategies beyond the aforementioned specialized therapies are absent. For this reason, innovation and improvement in the non-specialized mental health care for patients with severe BPD appears both necessary and useful. Obviously the areas in need of such innovation and improvement s must first be identified and, in this paper, the predominant problems encountered in community mental health care for patients with severe BPD are thus outlined. Our aim in doing this, moreover, is to answer the following three research questions.

1) What are the problems in community mental health care for patients with severe BPD that do not fit into specialized treatments?

2) Which of the problems identified are judged to be most urgent by professionals?

3) Which of the problems identified are judged by professionals as most amenable to improvement via professional intervention?

\section{Methods}

Design

A four-phase Delphi procedure was conducted to elicit and prioritize the views of a group of national experts on severe
BPD ( $n=8)$. The Delphi method is often used to explicate tacit knowledge and reach consensus on little-researched topics (Jones and Hunter 1995; Hasson et al. 2000). We modified the first round in the procedure with the use of a focus group to generate possibly relevant items, followed by a thematic analysis of the focus group discussion, as opposed to the anonymous generation of items. The second round consisted of participants' validation of the items derived from the initial analyses, and the third and fourth rounds involved participants' scoring of the items. The number of rounds was preset, and we did not encounter outcomes that necessitated design changes. The first round involved a face-to-face group meeting; the other rounds all involved correspondence via e-mail. BPD was defined according to the DSM-IV criteria (APA 1994), and severity of the BPD was established via selection of the Delphiparticipants from settings in which predominantly low functioning BPD-patients are known to be treated.

\section{Participants}

Experts with expertise on the treatment of patients with a BPD and at least some experience with the specialized treatment of such patients, but working in a general setting such as a community mental health center or general psychiatric department of a hospital, were intentionally approached. The expert group was composed of eight mental health professionals from different disciplines, different treatment locations, and different educational backgrounds (Table 1). To be included in the present study, the experts had to meet the following two criteria: (1) have at least 3 years of experience working with the target patient group and (2) either be employed at a nationally recognized center of expertise or a nationally recognized expert in terms of publications, lectures, and academic excellence. The experts were selected via a search of the recent literature on BPD and consultation with key figures at nationally recognized centers of expertise. The sample size of eight was considered adequate as the group of experts was (1) sufficiently homogeneous to focus on the same patients (i.e., community mental health experts on BPD) and (2) sufficiently heterogeneous to allow variation in the identification and scoring of problems (i.e., represented different disciplines, treatment locations, and educational backgrounds; Powell 2003).

\section{Procedure}

In the first round of the Delphi procedure, the focus group was interviewed to elicit as much information as possible on the problems encountered in the community mental health care for patients with severe BPD. Interaction 
Table 1 Characteristics of expert group

\begin{tabular}{ll}
\hline Characteristics & Number of experts \\
\hline Age & - \\
$<30$ & - \\
$30-39$ & 6 \\
$40-49$ & 2 \\
$50-59$ & - \\
$59>$ & \\
Sex & 4 \\
F & 4 \\
M & \\
Profession & 4 \\
Mental health nurse & 2 \\
Psychiatrist & 2 \\
Psychologist & \\
Treatment location & \\
Outpatient & 4 \\
Inpatient & 2 \\
Day treatment & 2 \\
Educational setting & \\
Non-academic & 6 \\
Academic & 2 \\
\hline
\end{tabular}

between the members of the focus group was stimulated as this was judged useful and necessary to illuminate this rare topic. It was expected that more varied results would be attained from the focus group interview and discussion than from individual interviews and/or questionnaires (see also Kitzinger 1995). The second author moderated the focus group discussion while the first author monitored content and asked for clarification as deemed necessary. A neutral space not affiliated with a psychiatric facility was used for the focus group discussion, which lasted $95 \mathrm{~min}$. The focus group interview items were prepared on the basis of a review of the relevant literature (Koekkoek et al. 2006) but were mostly introduced by the experts themselves in response to the introductory question of "What are the main problems in the psychiatric care for patients with a severe BPD?". The experts were asked to report on both their own personal experiences with such patients and also those of fellow professionals at their workplace. During the initial brainstorming phase of the group interview, the primary researcher noted all of the problems identified and divided them into five categories on which the experts agreed. During the second phase of the interview, the experts further elaborated upon the problems and provided concrete examples. The focus group interview was audiotaped, transcribed, and manually coded for thematic analysis (Joffe and Yardley 2004) using qualitative data analysis software (Kuckartz 2001). The initial coding structure was based upon the five categories of problems identified in the group interview. The research team discussed this code structure, re-analyzed the data descriptively, and produced a summary and list of specific problems.

In the second round of the Delphi procedure, the initial summary and list of problems were sent to all of the participating experts for validation. All of the experts returned the list with comments, which were then discussed by the research teams. The result was a final list of 36 problems.

In the third round of the Delphi procedure, the group of eight experts was asked to rate the 36 problems with regard to urgency ("To what extent do you judge this to be an urgent problem in the daily psychiatric care for severe BPD patients?") and their estimated changeability ("To what extent do you judge this problem to be amenable to improvement via a best-practice program?"). The experts responded using a seven-point Likert scale. The results were analyzed using SPSS (version 15); the data were treated as interval data; and the mean group scores and standard deviations were calculated. Those problems with a standard deviation larger than 1.5 were judged to have a lack of consensus. Those experts whose scores differed substantially ( $>1.5$ points) from the group mean were then, in keeping with the Delphi procedure, asked to elucidate their scores. For each of the relevant items, the comments of the experts were then summarized.

In the fourth and final round of the Delphi procedure, the experts were again given the list of 36 problems and also the summary of statements provided in round three. They were asked to reconsider their initial scores in light of the comments provided and provide a new score when judged to be relevant or necessary. The definitive group means and standard deviations were next calculated for the 36 problems identified by the group of experts.

\section{Conflict of Interest}

The authors declare that all certify responsibility, that none have a known conflict of interest, and that this manuscript has not been submitted to any other journal.

\section{Results}

After the third round of the Delphi procedure, a lack of consensus was observed for 14 of the 72 urgency and changeability judgments regarding the 36 problems identified by the experts. After the fourth round, a lack of consensus was still observed for 9 judgments. The scores on urgency and changeability are summarized with the highest scoring on urgency ranked first (Table 2), using the five categories derived at during the interview (patient-related, professional-related, interaction-related, social system- 
related and mental health care-related problems). In the following sections, these categories will be used to describe the quantitative results. Given that we considered the urgency score to be most important, the problems will be discussed according to their degree of urgency in the following. The problem's rank order is indicated in parentheses. A lack of consensus is indicated by a pound sign (\#) following the problem's rank order. Where relevant or markedly different, changeability scores are also exemplified.

\section{Patients}

The largest problem, according to the experts, is the difficulties that the patients have with attachment (1) to people in general and professionals in particular. This is typical for all patients with a BPD but more pronounced in the severely disordered subgroup. The attachment problem is not limited to the dyadic treatment relationship, but is more widespread. The patient literally does not attach to any professional and drifts, thus, through the health care system with no continuity of care as a consequence. Patients have a hard time trusting a therapist and may only do so after quite some time, which professionals tend to underestimate.

These people really need a secure attachment, they fight it for a year, claim help and then reject it again.

But if one succeeds in breaking that pattern, one can really mean something. (psychologist)

On the other hand, a dependency relationship may be perceived as dangerous in community mental health care as many patients become long-term users that lay a large claim on scarce resources, according to the experts (2).

Relapse (3) is also perceived to be a major problem as this typically brings considerable turmoil (e.g., the cancellation of regular appointments, need for after-hours crisis intervention, disagreement between professionals, hospitalization). And such turmoil obviously interferes with the development of a long-term commitment to treatment. The experts perceive the urgency of the problems (4), complexity of the problems (5), and large amount of problems (7) to be almost equally important stumbling blocks. Many of the problems are of a social nature such as having no house, no work, little money, or too few people to rely upon-due in part to attachment problems. All of these problems also make treatment quite difficult. Suicidality (6) and personality problems (8) are judged, however, to be relatively less urgent; the experts mention them as problems intrinsic to their work with such patients.

The rank ordering of the changeability of the patient problems was markedly different than the rank ordering of their urgency according to the experts. Urgent patient problems (8), complex patient problems (7), and patient dependency (6) ranked lowest with regard to changeability although the absolute differences between the various problems were quite small (see Table 2). In contrast, patient personality problems were ranked lowest for urgency (8) and high for changeability (2).

\section{Professionals}

The most prominent problem in this category of problems is a pessimistic attitude on the part of professionals towards patients and treatment (1). Compared to therapists specialized in the treatment of BPD, community mental health professionals rarely see patients improve under their care. Belief in the possibilities of success is thus limited, and many professionals report being wary of becoming emotionally involved.

Many find it [building a therapeutic alliance] fright-

ening, I think. Some 10 years ago the general opinion

was that these patients should be kept at distance, to

not let them get attached to you. (psychologist)

The experts think that the aforementioned opinion is still prevalent in community mental health care today. Patients with severe BPD are perceived as unpredictable and as individuals to whom one cannot relate. And from here, it is but a small step towards "blaming the patient."

What I have noticed repeatedly is that somewhere along the way, when treatment proves to be disappointing, the patient increasingly gets blamed. (psychiatrist)

The experts attribute this process to a lack of general and specific therapeutic skills for the handling of patients with a severe BPD $(2,3)$. Professionals may consider suicidality intrinsic to such patients, but few are able to handle it very well (2). A fearful attitude and restrictive interventions can be particularly problematic because they only reinforce a crisis or relapse on the part of patients. The special dynamics of chronic and recurrent suicidality may also be poorly understood in services that are mostly aimed at psychotic or depressed patients who are acutely suicidal.

\section{Interaction}

According to the experts, the main problem in the interaction category of problems is closely related to the problems in the patient and professional categories: The patient is considered able but unwilling to behave differently (1). The patient simply does not "stick to the rules" (e.g., comply with treatment recommendations, show a motivation to get better) while psychotic patients, in contrast, are not expected to do such. When a patient with a severe BPD is thus perceived as wasting the professional's time and energy (2), an incongruence in the treatment 
Table 2 Urgency and changeability scores for five categories of problems identified in the community mental health care for patients with severe borderline personality disorder

\begin{tabular}{|c|c|c|c|c|c|}
\hline \multirow[t]{2}{*}{$\mathrm{Nr}$} & \multirow[t]{2}{*}{ Category and problem } & \multicolumn{2}{|c|}{ Urgency } & \multicolumn{2}{|c|}{ Changeability } \\
\hline & & Mean & SD & Mean & SD \\
\hline \multicolumn{6}{|c|}{ I: Patients } \\
\hline 1 & Attachment disorders & 6.13 & .64 & $5.00(3)$ & 1.20 \\
\hline 2 & Dependency & 5.75 & .71 & $4.75(6)$ & 1.67 \\
\hline 3 & Relapses & 5.38 & .92 & $5.50(1)$ & 1.07 \\
\hline 4 & Urgent problems & 5.38 & 1.06 & $4.63(8)$ & .74 \\
\hline 5 & Complex problems & 5.13 & 1.36 & $4.75(7)$ & 1.83 \\
\hline 6 & Suicidality & 4.88 & 1.13 & $4.88(4)$ & .99 \\
\hline 7 & Large amount of problems & 4.75 & 1.28 & $4.75(5)$ & 1.49 \\
\hline 8 & Personality problems & 3.88 & 1.46 & $5.13(2)$ & 1.25 \\
\hline \multicolumn{6}{|c|}{ II: Professionals } \\
\hline 1 & Pessimistic attitude & 5.50 & 1.07 & $4.88(1)$ & 1.55 \\
\hline 2 & Fearful attitude with regard to suicidality & 5.38 & 0.92 & $4.63(2)$ & 1.12 \\
\hline 3 & Limited general therapeutic skills & 5.25 & 1.04 & $4.63(3)$ & 1.41 \\
\hline \multicolumn{6}{|c|}{ III: Interaction } \\
\hline 1 & Patient is able but unwilling (in view of professional) & 5.75 & 1.04 & $5.13(2)$ & 1.25 \\
\hline 2 & Interference with time/agenda of professional & 5.38 & .74 & $5.13(1)$ & 1.13 \\
\hline 3 & Powerlessness (in patient and professional) & 5.00 & .93 & $4.75(5)$ & 1.58 \\
\hline 4 & Feeling pressured (in professional) & 4.88 & 1.46 & $4.75(3)$ & 1.39 \\
\hline 5 & Undertreatment due to demoralization & 4.75 & 1.39 & $4.75(4)$ & 1.39 \\
\hline 6 & Demoralization (in patient and professional) & 4.63 & 1.60 & $4.38(7)$ & 1.85 \\
\hline 7 & Lack of appreciation/success (in patient) & 4.38 & 1.19 & $4.38(6)$ & 1.06 \\
\hline 8 & Lack of congruence in expectations & 3.75 & 1.04 & $4.25(8)$ & 1.39 \\
\hline \multicolumn{6}{|c|}{ IV: Family, social system, and society } \\
\hline 1 & Poor relationship between family of patient and professionals & 4.88 & .99 & $4.50(1)$ & 1.07 \\
\hline 2 & Poor parental functioning on part of patient & 4.88 & 1.36 & $4.50(2)$ & 1.41 \\
\hline 3 & Lack of family support & 4.88 & 1.46 & $4.38(4)$ & 1.77 \\
\hline 4 & Poor social functioning & 4.38 & 1.06 & $4.38(3)$ & 1.06 \\
\hline 5 & Limited role of patient in family & 4.38 & 1.30 & $3.88(5)$ & .99 \\
\hline \multicolumn{6}{|c|}{$V:$ Mental health system } \\
\hline 1 & Lack of organizational support & 5.63 & 1.06 & $5.50(1)$ & .93 \\
\hline 2 & Lack of structured treatment & 5.50 & 1.07 & $5.38(4)$ & 1.19 \\
\hline 3 & Lack of reflection & 5.50 & 1.20 & $4.75(12)$ & 1.75 \\
\hline 4 & No view on problems and treatment & 5.25 & .89 & $5.13(9)$ & 1.25 \\
\hline 5 & Limited cooperation between professionals & 5.13 & 1.13 & $5.25(6)$ & 1.04 \\
\hline 6 & Only "pampering and dithering" & 5.13 & 1.36 & $5.13(10)$ & 1.36 \\
\hline 7 & Lack of treatment agreements & 5.00 & 1.41 & $4.88(11)$ & 1.64 \\
\hline 8 & Lack of consistent treatment & 4.88 & .99 & $5.38(2)$ & .91 \\
\hline 9 & Lack of long-term treatment & 4.88 & 1.55 & $5.38(5)$ & 1.30 \\
\hline 10 & Lack of intensive treatment & 4.88 & 1.96 & $5.25(7)$ & 1.39 \\
\hline 11 & High but inefficient use of services & 4.75 & 1.28 & $5.38(3)$ & 1.06 \\
\hline 12 & Diffusion of responsibility & 4.75 & 1.39 & $5.25(8)$ & 1.49 \\
\hline
\end{tabular}

expectations of the patient and the professional becomes apparent (8) and the professional can easily start to perceive the patient as "bad" as opposed to "mad."
In a crisis intervention center, patients with a psychosis were seen as not accountable and in need of support. Borderline patients, however, were considered 
theatrical, posing, and in need of punishment. (psychologist)

Severe borderline patients, according to the experts, can make professionals feel pressured to intervene (4). Given the known lack of treatment appreciation and success (7), however, the professionals feel quite powerless (3) and may even feel demoralized (6\#). The combination of powerlessness and the blaming of the patient for any lack of progress may result in non-therapeutic behaviors on the part of the professional such as irritation, anger, and even aggression. Less overt but equally destructive is the reduction of the therapeutic encounter to doing as little as possible and simply hoping that a crisis does not arise. Further referral of the patient without substantial justification of the reasons for doing this is another example of really doing nothing.

Professionals have many strategies to do completely nothing in therapeutic encounters with the patient. (psychiatrist)

Such undertreatment (5) may occur more easily in the more hectic and medical atmosphere of a community mental health center, where acutely psychotic patients often take priority, than in a specialized therapy. Paradoxically, such undertreatment may also result in the very dependency that professionals try to avoid. That is, subtherapeutic treatment dosages do not solve problems but, rather, make patients come back for more, according to experts. Some of the experts are particularly critical of the apparent denial that can be seen to occur in several settings of the long-term nature of the problems of the patient with a severe BPD. That is, a rather naïve and overly "optimistic" attitude characterizes professionals who rapidly discharge such patients. According to the experts, in fact, such optimism is simply "therapeutic nihilism" disguised as optimism.

\section{Family, Social System, and Society}

The contact between borderline patients and their families is often severely disturbed. Years of mutual misunderstanding have eroded any natural provision of support, according to the experts.

As far as I know these patients, they have only one support system left and that is mental health care. (psychiatrist)

Many relatives have high expectations of the mental health system but are disappointed over time, which also results in a poor relationship between the family of the patient and professionals (1). The social functioning of the patient group itself is very poor. Both as parents (2), family members ( $3 \& 5$ ), and citizens (4), patients with a severe BPD tend to have limited skills. Experts therefore worry, in particular, about the children being raised in a family with a severe borderline patient and struggle with the question of if and when to intervene.

One is not allowed to approach it, to touch it, nothing is wrong with the children [according to the patient]. (mental health nurse)

And meanwhile, child protective services are requesting information from you. (psychiatrist)

\section{Mental Health System}

The largest number of problems reported by the experts, by far, lies within the mental health system with the lack of organizational support (1) constituting a major issue. According to the experts, many of the conditions necessary for the proper care of patients with a severe BPD are simply not met in community mental health. Structured treatment (2) with additional time for inter-collegial reflection on personal feelings and attitudes (3) is often absent. Without a coherent view of the specific features of the disorder and its treatment (4), operationalized in the form of a concrete treatment plans (7), heated debate frequently occurs between professionals with regard to patients (5) and the day-to-day care for patients run amuck.

Often a consistent treatment approach is no longer maintained (...) The track is left and all that remains

is 'staying present' [with the patient]. (psychologist)

The lack of treatment consistency (8) and continuity (9\#) (i.e., long-term care) is perceived to be particularly problematic for severe borderline patients who are thought to be even more sensitive to such disturbances than other psychiatric patients. The high but inefficient use of the services of several agencies by patients (11) contributes further to this lack of continuity, the diffused nature of the treatment being offered by professionals, and responsibility for treatment (12). The experts believe that these disruptions are also-at least in part-caused by the constantly changing health care policies and financing but may nevertheless also have to do with the therapeutic setting itself.

To which extent is mental health care capable of offering consistency and are professionals allowed to work with patients for prolonged periods of time? (mental health nurse)

In community mental health care, moreover, there are very few options available between the intensive treatment that some-but not all-experts think is necessary for patients with a severe BPD (10\#) and doing as little as possible, which is referred to as "pampering and dithering" 
(6). Just as for the patient-related category of problems, marked differences between the rank ordering of the problems with regard to urgency and changeability were also present for the category of problems related to the mental health system. The lack of organizational support was considered not only urgent (1) but also changeable (1). In contrast, the lack of opportunities for professional reflection and consultation was judged to be a relatively urgent problem but difficult to change (12). Conversely, the experts believed that a problem with relatively little urgency, namely that of high service use (11), could be easily altered (3).

\section{Discussion}

The main results of this research show in detail that the community mental health care for patients with severe BPD is perceived to be particularly difficult. Not only patient and professional variables but also organizational variables are found to differ substantially from those for specialized treatment. When these differences are taken into account, improvement of the community mental health care for patients with a severe BPD appears to be possible. Some eminent researchers on specialized therapies were present in our Delphi panel of experts and noted the limitations of these therapies and the need to improve "care as usual." The data show not only the urgency of several of the problems noted by the experts for the non-specialized mental health care offered to patients with severe BPD but also the optimism of the experts with regard to the possibilities for improvement as reflected by many equally high changeability scores. Some notable exceptions to this pattern of agreement between the urgency and changeability scores were also nevertheless apparent in the categories of problems relating to the patient and the mental health system. Some problems were judged to be urgent but difficult to change and vice versa. Nonetheless, patient relapse, the attributions of professionals, organizational support, and the provision of more structured and consistent long-term treatment all received changeability scores in the upper part of the scale, which indicates areas for potential improvement. In the following, we will discuss these findings in greater detail after consideration of some possible limitations on this exploratory study.

One possible limitation on the present study is the small number of experts involved. The Delphi procedure, however, is known to be suited for use with small samples-particularly when the sample has been selected purposefully. In the present study, the sample was formed by consulting with and inviting important stakeholders, which resulted in a sample that was both diverse (i.e., involved different professions, ages, sexes and backgrounds) and specific (i.e., all participants were community mental health experts on BPD). A weakness of the Delphi procedure is its strong emphasis on consensus and the suggestion that the outcomes are the only possible truth. We therefore decided against the use of cut-off points for the interpretation of the mean scores and to interpret the results both quantitatively and qualitatively instead. Five qualitative categories of problems agreed upon by the experts and researchers guided the analyses of the results. Although some of the problems reported by the experts could concern more than one category, which indicates the complexity of the issues involved, we opted to maintain the initial classification of the problems as agreed upon by the participants during the first round of the Delphi procedure (i.e., interview) and confirmed by them during the second round (i.e., expert check). Generalization of the results may also be limited to the extent that differences exist in the accessibility and financing of mental health care systems across the world. Given the scarce number of studies available, however, we can tentatively conclude that the nonspecialized care for patients with severe BPD may be problematic in most highly industrialized countries (see also Koekkoek et al. 2010).

Our first major finding is that severe borderline patients appear to have a greater number of and qualitatively different problems than other borderline patients. Several of the patient-related problems mentioned by the experts (e.g., dependency, presentation of numerous urgent and complex problems) are not equivalent to the DSM criteria for the diagnosis of such a disorder. In other words, the problems that experts encounter with severe borderline patients can only be attributed in part to the core symptoms of the disorder. This may be typical of the borderline patient in community mental health care; that is, the many complicating problems reported by such patients may obscure the core symptoms of the BPD. For instance, social functioning and support are both quite limited. Similar problems are reported in studies of the care for patients with chronic depression; "difficult" patients are reported to be highly dependent and to suffer from additional complex, social problems (Koekkoek et al. 2008). With the exception of two problems, all of the patient-related problems were assigned relatively lower changeability scores than urgency scores, which showed the experts to indeed consider these patients severely ill and hard-to-treat.

A second major finding is that the professionals in community mental health care differ from those in specialized therapies. The professionals in community mental health care do not have a solid understanding of BPD and its possible causes to fall back on; nor do they have a coherent treatment philosophy. For this reason, any difficult behavior on the part of the patient may often be interpreted as a willful attempt to undermine treatment. This tendency to interpret behavior differently depending 
upon a patient's diagnosis is congruent with the relevant research literature (e.g., Markham and Trower 2003; Gallop et al. 1989; Lewis and Appleby 1988). Axis I disorders such as depression and psychosis have been shown to evoke a more understanding and caring reaction than an Axis II disorder such as BPD-particularly in settings where the former appear more often than the latter. However, this particular problem along with some other professional-related and interaction-related problems was assigned a high changeability score, which shows the experts to believe that training is necessary and possible: that is, the attitudes of professionals towards patients with a severe BPD can and should be improved.

A third major finding is that the organization of community mental health care and the problems associated with this care clearly differ from that of specialized therapies. Today, community mental health care is often not arranged for the intensive treatment of patients such as those with a severe BPD. The arrangement of community mental health care is not based upon a clear view on BPD, does not provide structured treatment for the disorder, does not have the required coherence, and does not offer sufficient support for the professionals involved. Without clear principles to guide them, professionals may find themselves lost with these patients. Uncertainties may reinforce difficulties in the therapeutic relationship, and unpopular decisions such as diminishing the frequency of a care contact may be based upon the personal choices of the professionals rather than a treatment framework. Patients may thus see professionals as individuals who make more or less arbitrary decisions (i.e., decisions based upon personal opinions), which may-in fact-be true when an overall view of the disorder and its treatment is absent. Both "blaming the patient" for not being like other longterm psychiatric—often psychotic—patients and "blaming the system" for not offering sufficient structure and support will be the result. Unlike the other categories of problems, the problems reported with respect to the mental health care system were assigned relatively high changeability scores by the experts in our study, showing that systemic changes are possible and required to improve the care for patients with severe BPD.

As things stand today, severe borderline patients and community mental health care are often "mismatched." Given that a "match" between these patients and specialized therapies neither exists, community mental health care may be the only available safety net for severe BPD patients. In fact, the best practical and social support for poorly functioning patients can be found in communitybased rehabilitation care and not intrapersonal or interpersonal psychotherapies while the latter interpersonal psychotherapeutic treatment context is best suited for dealing with the maintenance of a positive therapeutic alliance. In daily practice, severe BPD patients do not receive either of these treatments as they do not qualify for either. Nonetheless, specialized psychotherapy could lower the threshold for patients with numerous social, motivational, and other problems while community care could increase its focus on the attitudes of the professionals involved, the therapeutic alliance, and the organization of the necessary care. Given that community care is often the safety net for other forms of mental health treatment, our preference is for this option.

With regard to policy, patients with a severe BPD should probably be considered a special subgroup for community mental health care. Professionals working with these patients may need special training (e.g., on motivational techniques, on techniques to establish and maintain a therapeutic alliance), more direct support (e.g., supervision), more indirect support (e.g., care guidelines, a theoretical framework for understanding patient behavior), and greater organizational structure (e.g., shared and possibly smaller case loads, easier access to specialists). Explanations and effective care strategies from both long-term rehabilitation programs and specialized therapies may be combined into an intervention program specifically aimed at this patient group. General care strategies such as crisis prevention, needs assessment, mutual goal setting, and active case-management can be combined with more specific care strategies such as an interpersonal focus, analysis, and techniques for the management of self-destructive behaviors in addition to the aforementioned organizational changes.

\section{Conclusions}

This research highlights some of the specific problems encountered in the community mental health care for patients with a severe BPD. The aim of such care should be, on the one hand, to motivate and prepare patients for specialized treatment. On the other hand, long-term care with realistic goals and adequate support for cases of clear social dysfunction must be developed as well. The goal of our future research is therefore to construct a best-practice program that is easy to use by non-specialized, non-psychotherapeutic professionals in community mental health care.

Open Access This article is distributed under the terms of the Creative Commons Attribution Noncommercial License which permits any noncommercial use, distribution, and reproduction in any medium, provided the original author(s) and source are credited.

\section{References}

Bateman, A., \& Fonagy, P. (1999). Effectiveness of partial hospitalization in the treatment of borderline personality disorder: A randomized controlled trial. The American Journal of Psychiatry, 156(10), 1563-1569. 
Bateman, A., \& Fonagy, P. (2001). Treatment of borderline personality disorder with psychoanalytically oriented partial hospitalization: An 18-month follow-up. The American Journal of Psychiatry, 158(1), 36-42.

Bateman, A., \& Fonagy, P. (2008). 8-Year follow-up of patients treated for borderline personality disorder: Mentalization-based treatment versus treatment as usual. The American Journal of Psychiatry, 165(5), 631-638.

Bender, D. S., Dolan, R. T., Skodol, A. E., Sanislow, C. A., Dyck, I. R., McGlashan, T. H., et al. (2001). Treatment utilization by patients with personality disorders. The American Journal of Psychiatry, 158(2), 295-302.

Clarkin, J. F., Levy, K. N., Lenzenweger, M. F., \& Kernberg, O. F. (2007). Evaluating three treatments for borderline personality disorder: A multiwave study. The American Journal of Psychiatry, 164(6), 922-928.

Comtois, K. A., Russo, J., Snowden, M., Srebnik, D., Ries, R., \& Roy-Byrne, P. (2003). Factors associated with high use of public mental health services by persons with borderline personality disorder. Psychiatric Services, 54(8), 1149-1154.

Gallop, R., Lancee, W. J., \& Garfinkel, P. (1989). How nursing staff respond to the label "borderline personality disorder". Hospital Community Psychiatry, 40(8), 815-819.

Giesen-Bloo, J., van Dyck, R., Spinhoven, P., van Tilburg, W., Dirksen, C., van Asselt, T., et al. (2006). Outpatient psychotherapy for borderline personality disorder: Randomized trial of schema-focused therapy vs transference-focused psychotherapy. Archives of General Psychiatry, 63(6), 649-658.

Greenwood, N., Chisholm, B., Burns, T., \& Harvey, K. (2000). Community mental health team case-loads and diagnostic casemix. Psychiatric Bulletin, 24, 290-293.

Hasson, F., Keeney, S., \& McKenna, H. (2000). Research guidelines for the Delphi survey technique. Journal of Advanced Nursing, 32(4), 1008-1015.

Hunter, M., Jadresic, D., Blaine, A., Clancy, L., Leyshon, N., McDonald, E., et al. (2002). Two weeks in the life of a community mental health team: A survey of case-mix and clinical activity in the north-west of Sheffield. Psychiatric Bulletin, 26(1), 9-11.

Joffe, H., \& Yardley, L. (2004). Content and thematic analysis. In D. Marks \& L. Yardley (Eds.), Research methods for health and clinical psychology (pp. 56-68). London: Sage.

Jones, J., \& Hunter, D. (1995). Consensus methods for medical and health services research. BMJ, 311(7001), 376-380.

Keown, P., Holloway, F., \& Kuipers, E. (2002). The prevalence of personality disorders, psychotic disorders and affective disorders amongst the patients seen by a community mental health team in London. Social Psychiatry and Psychiatric Epidemiology, 37(5), 225-229.
Kitzinger, J. (1995). Qualitative research. Introducing focus groups. BMJ, 311(7000), 299-302.

Koekkoek, B., van Meijel, B., \& Hutschemaekers, G. (2006). "Difficult patients" in mental health care: A review. Psychiatric Services, 57(6), 795-802.

Koekkoek, B., van Meijel, B., Schene, A., \& Hutschemaekers, G. (2008). Clinical problems in the long-term care of patients with chronic depression. Journal of Advanced Nursing, 62(6), 689-697.

Koekkoek, B., van Meijel, B., \& Hutschemaekers, G. (2010). Community mental health care for patients with severe personality disorder: A narrative review. Psychiatric Bulletin (in press).

Kuckartz U. (2001). MAXqda qualitative data analysis introduction. Berlin: Verbi Software. Consult. Sozialforschung.

Lesage, A. D., \& Cope, S. J. (1988). Personality disorders in long term non-psychotic patients. Social Psychiatry and Psychiatric Epidemiology, 23(3), 158-162.

Lewis, G., \& Appleby, L. (1988). Personality disorder: The patients psychiatrists dislike. The British Journal of Psychiatry, 153, 44-49.

Lieb, K., Zanarini, M. C., Schmahl, C., Linehan, M. M., \& Bohus, M. (2004). Borderline personality disorder. Lancet, 364(9432), 453-461.

Linehan, M. M., Armstrong, H. E., Suarez, A., Allmon, D., \& Heard, H. L. (1991). Cognitive-behavioral treatment of chronically parasuicidal borderline patients. Archives of General Psychiatry, 48(12), 1060-1064.

Linehan, M. M., Comtois, K. A., Murray, A. M., Brown, M. Z., Gallop, R. J., Heard, H. L., et al. (2006). Two-year randomized controlled trial and follow-up of dialectical behavior therapy vs therapy by experts for suicidal behaviors and borderline personality disorder. Archives of General Psychiatry, 63(7), 757-766.

Markham, D., \& Trower, P. (2003). The effects of the psychiatric label 'borderline personality disorder' on nursing staff's perceptions and causal attributions for challenging behaviours. The British Journal of Clinical Psychology, 42(Pt 3), 243-256.

Nehls, N., \& Diamond, R. J. (1993). Developing a systems approach to caring for persons with borderline personality disorder. Community Mental Health Journal, 29(2), 161-172.

Paris, J. (2002). Chronic suicidality among patients with borderline personality disorder. Psychiatric Services, 53(6), 738-742.

Pomeroy, J. C., \& Ricketts, B. (1985). Long-term attendance in the psychiatric outpatient department for non-psychotic illness. The British Journal of Psychiatry, 147, 508-516.

Powell, C. (2003). The Delphi technique: Myths and realities. Journal of Advanced Nursing, 41(4), 376-382.

Tyrer, P. (2005). The problem of severity in the classification of personality disorder. Journal of Personality Disorders, 19(3), 309-314. 